\title{
Pinus thunbergii bark extract rich in flavonoids promotes hair growth in dorsal skin by regulating inflammatory cytokines and increasing growth factors in mice
}

\author{
YOUNG HER ${ }^{1 *}$, TAE-KYEONG LEE ${ }^{2 *}$, HYEJIN SIM $^{3}$, JAE-CHUL LEE $^{3}$, DAE WON KIM $^{4}$, SOO YOUNG CHOI ${ }^{2}$, \\ JUN KEE HONG ${ }^{5}$, JI-WON LEE ${ }^{5}$, JONG-DAI KIM ${ }^{6}$, MOO-HO WON ${ }^{3}$ and SUNG-SU KIM ${ }^{5}$ \\ ${ }^{1}$ Department of Dermatology, Kangwon National University Hospital, Kangwon National University School of Medicine, \\ Chuncheon, Gangwon 24289; ${ }^{2}$ Department of Biomedical Science and Research Institute for Bioscience and Biotechnology, \\ Hallym University, Chuncheon, Gangwon 24252; ${ }^{3}$ Department of Neurobiology, School of Medicine, \\ Kangwon National University, Chuncheon, Gangwon 24341; ${ }^{4}$ Department of Biochemistry and Molecular Biology \\ and Research Institute of Oral Sciences, College of Dentistry, Gangnung-Wonju National University, Gangneung, \\ Gangwon 25457; ${ }^{5}$ Famenity Co., Ltd., Uiwang, Gyeonggi 16006; ${ }^{6}$ Division of Food Biotechnology, \\ School of Biotechnology, Kangwon National University, Chuncheon, Gangwon 24341, Republic of Korea
}

Received August 29, 2021; Accepted January 14, 2022

DOI: $10.3892 / \mathrm{mmr} .2022 .12616$

\begin{abstract}
Korean maritime pine bark (Pinus thunbergii) has been used as an alternative medicine due to its beneficial properties, including anti-inflammatory effects. To date, the anti-inflammatory and hair growth-promoting effects of Pinus densiflora bark extract have remained elusive. Therefore, in the present study, Pinus thunbergii bark was extracted with pure water $\left(100^{\circ} \mathrm{C}\right)$ and the extract was examined to determine its polyphenol and flavonoid content. C57BL/6 mice were used to assess the effects of the extract to promote hair growth. The extract (1, 2 and 4\%) was topically applied onto shaved dorsal skin and hair growth was observed for 17 days. A significant increase in hair growth was observed
\end{abstract}

Correspondence to: Professor Moo-Ho Won, Department of Neurobiology, School of Medicine, Kangwon National University, 1 Kangwondaehak-gil, Chuncheon, Gangwon 24341, Republic of Korea

E-mail: mhwon@kangwon.ac.kr

Dr Sung-Su Kim, Famenity Co., Ltd., D1009, 40 Imi-ro, Uiwang, Gyeonggi 16006, Republic of Korea

E-mail: mwwwu@hanmail.net

*Contributed equally

Abbreviations: AGA, androgenetic alopecia; DW, distilled water; HGPS, hair growth-promoting score; IGF-I, insulin-like growth factor I; PBE, Pinus thunbergii bark extract; ROD, relative optical density

Key words: anti-inflammatory cytokine, hair follicle, insulin-like growth factor I, Pinus thunbergii, pro-inflammatory cytokine, vascular epidermal growth factor with 2 and $4 \%$ extract. Based on this finding, the optimal dose of the extract for effective hair growth promotion was determined to be $2 \%$. The mechanisms of hair growth promotion were investigated via immunohistochemical analysis of changes in inflammatory cytokines and growth factors in the hair follicles following treatment with $2 \%$ extract. The treatment reduced the levels of TNF- $\alpha$ and IL-1 $\beta$, which are pro-inflammatory cytokines, while it enhanced the levels of IL-4 and IL-13, which are anti-inflammatory cytokines, in the hair follicles. In addition, elevated insulin-like growth factor I and vascular epidermal growth factor were detected in hair follicles following treatment. Based on these findings, it was suggested that the extract of Pinus thunbergii bark may be utilized for hair loss prevention and/or hair growth promotion.

\section{Introduction}

Although hair loss does not generally represent a serious health issue, it is a matter of interest as it may reduce confidence and lead to social anxiety, particularly in patients with alopecia, who manifest psychiatric symptoms, including depression, social phobia and anxiety, compared with normal individuals $(1,2)$. Hair loss in humans may be triggered by diverse factors, including mental stress, heredity and drug therapy (i.e., anti-thyroid agents and retinoids) $(2,3)$. Alopecia may be largely divided into the following categories: Androgenetic alopecia (AGA), alopecia areata and telogen effluvium (TE) (4). Therefore, the treatment and management of alopecia depends on the cause and type of alopecia $(2,3,5)$.

Certain studies have suggested that abnormal inflammatory response in the hair follicles may affect hair loss $(6,7)$. In addition, growth factors have a pivotal role in hair regrowth and elongation (8-10). Therefore, the modulation of inflammatory response by inflammatory cytokines and the 
enhancement of growth factors represent crucial approaches in the management of hair loss $(3,8,11)$.

The pine tree belongs to the family of Pinaceae and various parts of the pine tree, including cones, bark and needles, have been used as an alternative medicine for numerous diseases (12). Among the parts of the pine tree, the bark has been used as a traditional remedy and nutritional supplement $(13,14)$. In particular, the extract from the bark of pine trees grown near the seashore displays strong biological attributes. For instance, PineXol ${ }^{\circledR}$, a commercialized extract from Korean red pine (Pinus densiflora) bark, is used as an additive in health functional foods and cosmetics due to its anti-inflammatory, antioxidant and anti-lipogenic activities $(15,16)$. In addition, Pycnogenol ${ }^{\circledR}$, a standardized extract derived from the bark of French coastal pine (Pinus pinaster Aiton) has moisturizing properties and improves the elasticity of human skin by increasing hyaluronic acid and collagen type I (17). These maritime pine bark extracts contain abundant phenols, flavonoids and proanthocyanidins $(12,18)$.

To the best of our knowledge, no previous studies have reported hair growth promotion using the extract from the bark of Pinus thunbergii grown near the seashore. Therefore, the aim of the present study was to investigate whether rich phenols and flavonoids were contained in the Pinus thunbergii bark extract (PBE) and the effects of PBE on hair growth was investigated using C57BL/6 mice, which represent good animal models for the study of the hair cycle due to truncal pigmentation only during the anagen phase $(19,20)$. In addition, a previous study reported a subtle inflammatory response in hair follicles under normal conditions (21). Thus, it was investigated whether hair growth was promoted by moderating inflammatory responses after PBE application based on changes in pro- and anti-inflammatory cytokines. Furthermore, in the present study, PBE-induced changes in growth factors were assessed.

\section{Materials and methods}

Preparation of PBE. Pinus thunbergii growing next to the seashore of Gangneung (Republic of Korea) was gathered and its outer bark was harvested (specimen no., KNKA201106224024; Korea National Arboretum, Gyeonggi, Republic of Korea). The bark was washed with pure water and fully dried. Subsequently, using an M20 grinder (IKA), the dehydrated bark was pulverized into a fine powder. The powder $(10 \mathrm{~g} / \mathrm{kg})$ was extracted with pure water $(100 \mathrm{l})$ at $100^{\circ} \mathrm{C}$ for $24 \mathrm{~h}$ three times and the extract was then filtered using a filter paper (Whatman no. 1; Whatman Ltd.). The extract was then concentrated with a vacuum evaporator (N-12; Eyela Singapore Pte. Ltd.) and the evaporated extract was rapidly freeze-dried at $-55^{\circ} \mathrm{C}$ using a lyophilizer (FD8512; ilshin BioBase Co. Ltd.) and stored at $-20^{\circ} \mathrm{C}$. The extraction yield was $1.4 \%$.

\section{Qualitative analysis of $P B E$}

Total phenol content. The total phenol contents were determined by Folin-Ciocalteu's colorimetric method (22) using gallic acid as a standard for the calibration curve. The sample solution $(1 \mathrm{ml})$ was mixed with $10 \%$ Folin-Ciocalteu reagent $(1 \mathrm{ml})$ and $0.2 \% \mathrm{Na}_{2} \mathrm{CO}_{3}$ reagent $(1 \mathrm{ml})$ and incubated for $1 \mathrm{~h}$ in the dark. Using a microplate reader (Molecular Devices, LLC), the reaction was monitored by measuring the absorbance at $750 \mathrm{~nm}$ and the results were calculated $(\mathrm{y}=14.39 \mathrm{x}+0.0117$, $\left.\mathrm{R}^{2}=0.9968\right)$ as gallic acid equivalents (GAE).

Total flavonoid content. The total flavonoid contents were determined by the aluminum nitrate method (23) using quercetin as a standard for the calibration curve. The sample solution $(0.5 \mathrm{ml})$ was mixed with $95 \% \mathrm{EtOH}(1.5 \mathrm{ml}), 10 \%$ aluminum nitrate $(0.1 \mathrm{ml}), 1 \mathrm{M}$ potassium acetate $(0.1 \mathrm{ml})$ and distilled water (DW; $2.8 \mathrm{ml}$ ) and incubated room temperature for $30 \mathrm{~min}$. Using a microplate reader (Molecular Devices, LLC), the reaction was monitored by measuring the absorbance at $415 \mathrm{~nm}$ and the results were calculated $(\mathrm{y}=1.6385 \mathrm{x}+0.0382$, $\left.\mathrm{R}^{2}=1.0000\right)$ as quercetin equivalents $(\mathrm{QE})$.

Experimental animals. Male C57BL/6 mice (total $\mathrm{n}=40$; age, 7 weeks; body weight, $26 \pm 2 \mathrm{~g}$ ) were provided by the Experimental Animal Center of Kangwon National University (Chuncheon, South Korea). They were housed under conventional conditions at a temperature of $24^{\circ} \mathrm{C}$ and relative humidity of $\sim 55 \%$ with a 12-h light/dark cycle and ad libitum access to food and water. The mice used in this experiment were randomly grouped $(n=10$ in each group) as follows: i) Control group, which was treated with DW, and the ii) 1, iii) 2 and iv) $4 \%$ PBE groups, which were respectively treated with 1,2 and $4 \%$ PBE.

The experimental procedures using the experimental mice strictly adhered to the 'Current international laws and policies' of the 'Guide for the Care and Use of Laboratory Animals' (24) and the experimental protocol was approved by the Institutional Animal Care and Use Committee of Kangwon National University (Chuncheon, Republic of Korea; approval no., KW-200114-1; approval date, January 22, 2020). Humane endpoints were not established as the protocol of the current animal experiment did not cause any severe pain or stress.

Topical application of PBE and hair growth-promoting score (HGPS). In accordance with precedent studies by our group $(3,25)$, topical application with PBE was performed. In brief, the mice were shaved on their dorsal skin. PBE was dissolved in DW and three days after shaving, $200 \mu 1$ of DW or PBE $(1,2$ or $4 \%)$ was topically applied on the dorsal skin twice a day for 17 days.

The HGPS was recorded as described previously (8). In short, it was scored by observing the skin with the naked eye based on the criteria of hair growth stages as follows: 0 , pink-colored shaved skin; 1 , gray-colored shaved skin; 2, black-colored shaved skin; 3 , initiation of hair growth on the shaved skin; 4, completely grown hair on the shaved skin.

Tissue preparation for histological analysis. The histological sections of the dorsal skin were prepared according to a previously described method (25). In brief, at 17 days after the initiation of the topical application with DW and PBE (1, 2 and 4\%), the mice were deeply anesthetized by an intraperitoneal injection of pentobarbital sodium $200 \mathrm{mg} / \mathrm{kg}$ (JW Pharm. Co., Ltd.) (26). Under deep anesthesia, they were perfused with saline $(0.85 \% \mathrm{NaCl} w / \mathrm{v} ; \mathrm{pH} 7.4$; flow rate, $3.0 \mathrm{ml} / \mathrm{min}$ ) through the left ventricle. Subsequently, to fix 
Table I. Primary and secondary antibodies for immunohistochemical staining.

A, Primary antibodies

\begin{tabular}{lrl}
\hline Antibody & Dilution & Supplier (cat. no.) \\
\hline Rabbit anti-TNF- $\alpha$ & $1: 1,000$ & Abcam (ab6671) \\
Rabbit anti-IL-1 $\beta$ & $1: 200$ & Santa Cruz Biotechnology, Inc. (sc-12742) \\
Rabbit anti-IL-4 & $1: 200$ & Santa Cruz Biotechnology, Inc. (sc-12723) \\
Mouse anti-IL-13 & $1: 200$ & Santa Cruz Biotechnology, Inc. (sc-393365) \\
Rabbit anti-IGF-I & $1: 200$ & Santa Cruz Biotechnology, Inc. (sc-9013) \\
Mouse anti-VEGF & $1: 300$ & Abcam (ab69479) \\
\hline
\end{tabular}

B, Secondary antibodies

Antibody

Biotinylated horse anti-mouse IgG

Biotinylated goat anti-rabbit IgG
Dilution

Supplier (cat. no.)

$1: 250$

$1: 250$
Vector Laboratories, Inc. (BA-2000)
Vector Laboratories, Inc. (BA-1000)

IGF, insulin-like growth factor.

the skin tissue, they were perfused with $4 \%$ paraformaldehyde. Thereafter, their dorsal skin tissues were removed and the tissues were further fixed by soaking them in the same fixative at room temperature for $6 \mathrm{~h}$. The skin tissue was then embedded in a paraffin wax block for sectioning, and sections (8- $\mu \mathrm{m}$ thickness) were obtained using a microtome (Leica Microsystems).

$H \& E$ staining. To investigate changes in the epidermis and dermis, including hair follicles, following the topical application of DW and PBE, H\&E staining was performed as described previously $(3,27)$. The prepared sections were stained with Gill's hematoxylin solution (Merck KGaA) for 8 min followed by eosin Y solution (Merck KGaA) for 3 min. These sections were then dehydrated by immersing them in a dilution series of ethyl alcohol $(50,70,80,90,95$ and $100 \%$ for $1 \mathrm{~min}$, respectively) and then cleared with xylene for $5 \mathrm{~min}$. Finally, these stained sections were semipermanently preserved by mounting cover glasses with Canada balsam (Kanto Chemical Co., Inc.).

To compare histopathological changes in the dorsal skin between the groups, the digital images of the sections stained with H\&E were captured from 5 sections per animal with a light microscope (BX53; Olympus Corporation) connected to a PC monitor. The H\&E-stained hair follicles were counted using an array of $255 \times 255$ pixels corresponding to a tissue area of $250 \mu \mathrm{m}^{2}$.

Immunohistochemical staining. To examine alterations in inflammatory cytokines and growth factors in the dorsal skin tissues between the groups, immunohistochemical staining was performed in accordance with certain previous studies with minor modifications $(3,28)$. In brief, the tissue sections were reacted with $0.3 \%$ hydrogen peroxide for $20 \mathrm{~min}$ at room temperature. Subsequently, they were immersed in
$5 \%$ normal horse or goat serum (Vector Laboratories Inc.) for horse anti-mouse $\mathrm{IgG}$ and goat anti-rabbit $\mathrm{IgG}$ respectively at room temperature for $30 \mathrm{~min}$. Thereafter, these sections were immunoreacted with each primary antibody (Table I) at $4^{\circ} \mathrm{C}$ for $24 \mathrm{~h}$. Subsequently, they were reacted with the corresponding secondary antibody, as presented in Table I, at room temperature for $2 \mathrm{~h}$ and were then incubated in Elite avidin-biotin enzyme complex (Vector Laboratories, Inc.) at room temperature for $1 \mathrm{~h}$ at room temperature. Thereafter, the visualization of labeling was achieved using 3,3'-diaminobenzidine tetrahydrochloride solution (MilliporeSigma) for two min at room temperature. Finally, these sections were rinsed, dehydrated in a dilution series of ethyl alcohol (70, 80, 90, 95 and $100 \%$ ), cleared in xylene and mounted with coverslips and Canada balsam.

Immunoreactivity for each analyte protein in each group was evaluated using the relative optical density (ROD) of each immunoreactive structure according to a previously described method $(3,8)$. In brief, five sections per mouse were used and observed using a light microscope (BX53) from Olympus Corporation, as described in the chapter on H\&E staining. The image was captured in a corresponding tissue area of $140 \times 140 \mu \mathrm{m}^{2}$ and calibrated into an array of $256 \times 256$ pixels. Each immunoreactive structure was quantified as the ROD using a 0-255 gray scale system and software (ImageJ; v.1.46; National Institutes of Health). In this experiment, the ratio of the ROD was calibrated by setting the ROD of the control to $100 \%$.

Statistical analysis. Data obtained in this research were expressed as the mean \pm standard error of the mean. The present data were statistically analyzed using SPSS 18.0 software (SPSS, Inc.). Statistical significance was established using one-way ANOVA with a post hoc Bonferroni test for all pairwise comparisons in a multiple-group situation. The 


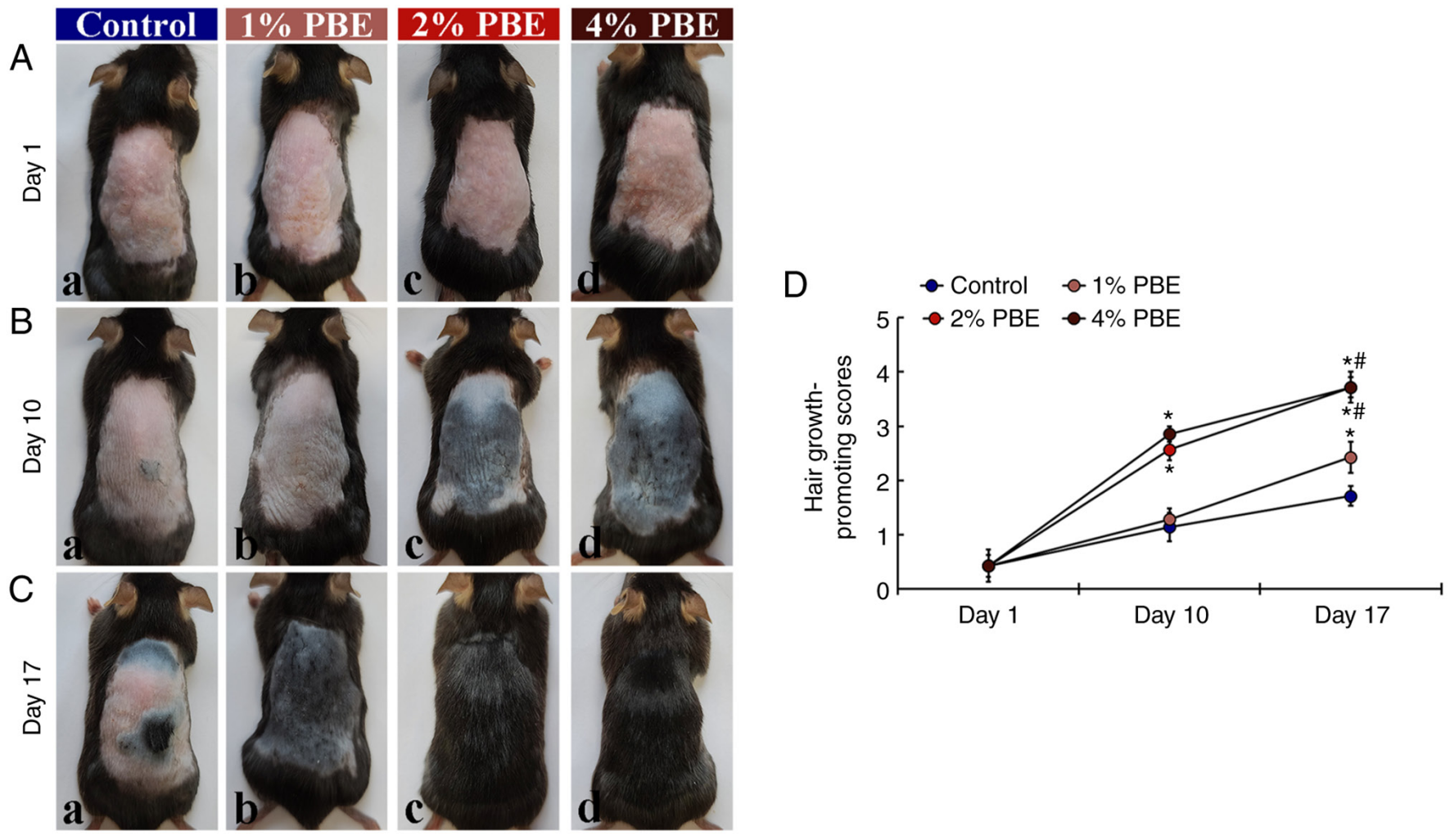

Figure 1. (A-C) Hair growth process in the dorsal skin of the (a) control, (b) $1 \%$, (c) $2 \%$ and (d) $4 \%$ PBE groups at (A) day 1, (B) day 10 and (C) day 17 . At 10 days, in the control and 1\% PBE groups, a pink-colored skin area is observed, whereas, at this time-point, in the 2 and $4 \%$ PBE groups, a gray- and black-colored skin area is present. Complete hair growth is observed at 17 days in the 2 and 4\% PBE groups. (D) Hair growth-promoting scores. The bars indicate the means \pm standard error of the mean ( $\mathrm{n}=10$ in each group). ${ }^{*} \mathrm{P}<0.05$ vs. control group; ${ }^{\sharp} \mathrm{P}<0.05$ vs. $1 \%$ PBE group. PBE, Pinus thunbergii bark extract.

Kruskal-Wallis followed by Dunn's test were used in order to statistically analyze the hair growth promoting scores. $\mathrm{P}<0.05$ was considered to indicate statistical significance.

\section{Results}

Total polyphenol and flavonoid contained in PBE. The total polyphenol and flavonoid content contained in PBE was determined to be $197.89 \pm 0.66 \mathrm{mg} \mathrm{GAE} / \mathrm{g}$ and $48.82 \pm 0.14 \mathrm{mg}$ $\mathrm{QE} / \mathrm{g}$, respectively, and the ratio of total flavonoid vs. total polyphenol was $0.2467 \pm 0.0015$ (data not shown).

Hair growth-promoting effect of PBE. First, hair growth in each group was evaluated by qualitative observation and scoring (Fig. 1). In the control group, pink-colored dorsal skin was apparent until 10 days of topical application of DW (Fig. 1A-a and B-a). In this group, gray- and black-colored dorsal skin with short hairs was sparsely present at 17 days of DW application (Fig. 1C-a). In the 1\% PBE group, the progress of hair growth at 10 days of $1 \% \mathrm{PBE}$ application was not different from that in the control group (Fig. 1A-b, B-b and D). At day 17, in this group, gray-colored dorsal skin was apparent and a significant progression of hair growth was observed compared with that in the control group (Fig. 1C-b and D). On the other hand, in the 2 and 4\% PBE groups, hair growth was significantly promoted at 10 days of application compared with that in the control group (Fig. 1B-c, B-d and D). In addition, at day 17, fully grown fur was detected in these two groups (Fig. 1C-c, C-d and D). Based on this observation, it was indicated that the most effective promotion of hair growth was accomplished by application of $2 \%$ PBE. Thus, the following histological investigations were performed with $2 \%$ PBE. Among the four groups, there was no site-specific allergic reaction and no change in body weight was observed after topical application of DW of PBE (1,2 and 4\%) (data not shown).

Effect of PBE on hair follicles. As presented in Fig. 2, the histology of the dorsal skin containing hair follicles in the PBE group was compared with that in the control group at 17 days of PBE treatment using H\&E staining (blue by hematoxylin and purple by eosin). In the control group, a typical morphology of the hair follicles was observed (Fig. 2A). In the PBE group, the stainability with $\mathrm{H} \& \mathrm{E}$ and cellular morphology in the hair follicles were not significantly different from those in the control group (Fig. 2B).

In the control group, the numbers of hair follicles and epithermal thickness $\left(8.1 / 250 \mu \mathrm{m}^{2}\right.$ and $16.6 \mu \mathrm{m}$, respectively) were not significantly different from those in the PBE group (7.9/250 $\mu \mathrm{m}^{2}$ and $16.7 \mu \mathrm{m}$; Fig. $2 \mathrm{C}$ and D, respectively). In addition, no infiltration of inflammatory cells was observed in the dorsal skin following topical application of DW of PBE in any of the groups (Fig. 2A and B).

Effect of PBE on pro-inflammatory cytokines. The effect of PBE on inflammatory cytokines was then assessed (Fig. 3). At 17 days of treatment, TNF- $\alpha$ immunoreactivity in the skin of the control group was principally detected in the hair follicles (Fig. 3A). In the PBE group, significantly 

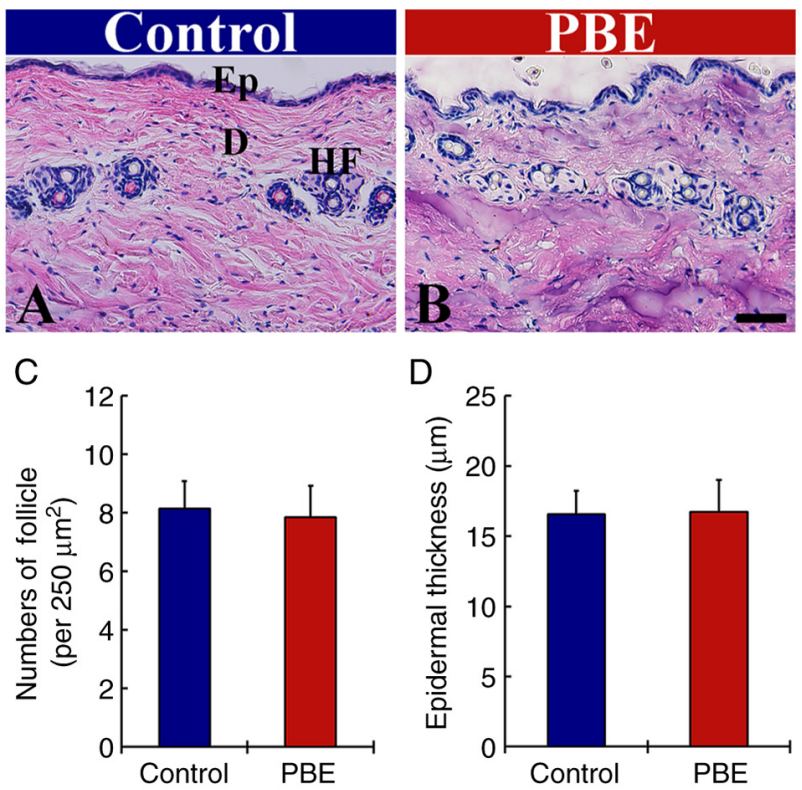

Figure 2. H\&E staining in the dorsal skin of (A) the control and (B) $2 \% \mathrm{PBE}$ groups at 17 days after the topical application of distilled water and $2 \%$ PBE respectively (scale bar, $50 \mu \mathrm{m}$ ). No significant difference was detected in their hair follicles (arrows) between the two groups. (C) Mean numbers of hair follicles and (D) mean thickness of epidermis. The bars indicate the means \pm standard error of the mean ( $\mathrm{n}=10$ in each group). Ep, epidermis; D, dermis; HF, hair follicle; PBE, Pinus thunbergii bark extract.

weakened TNF- $\alpha$ immunoreactivity (37.0\% vs. control group) was observed as compared with that in the control group (Fig. 3B and E).

In addition, strong IL-1 $\beta$ immunoreactivity was obtained in the hair follicles of the control group at 17 days of the treatment (Fig. 3C). In the PBE group, IL-1 $\beta$ immunoreactivity was significantly lower (49.7\% vs. control group) compared with that in the control group (Fig. 3D and F).

Effect of PBE on anti-inflammatory cytokines. The effect of PBE on anti-inflammatory cytokines was then assessed (Fig. 4). In the control group, immunoreactivity of IL-4 was fundamentally observed in the hair follicles at 17 days of treatment (Fig. 4A). In the PBE group, the immunoreactivity of IL-4 was significantly increased (149.6\% vs. control group) when compared with that in the control group (Fig. 4B and E).

In addition, the immunoreactivity of IL-13 in the control group was predominantly present in the hair follicles (Fig. 4C). In the PBE group, the immunoreactivity of IL-13 was significantly enhanced (192.8\% of the control group) as compared with that in the control group (Fig. 4D and F).

Effect of PBE on growth factors. The effect of PBE on growth factors was then assessed (Fig. 5). At 17 days of treatment, immunoreactivity of insulin-like growth factor (IGF)-I in the control group was weakly present in the hair follicles (Fig. 5A). In the PBE group, the immunoreactivity of IGF-I was significantly enhanced (280.2\% of control group) when compared with that in the control group (Fig. 5B and E).

In addition, the immunoreactivity of VEGF in the control group was fundamentally detected in the hair follicles
(Fig. 5C). In the PBE group, a significantly elevated immunoreactivity of VEGF (254.5\% of control group) was observed when compared with that in the control group (Fig. 5D and F).

\section{Discussion}

The results of the present study indicated that $\mathrm{PBE}$ contained polyphenols (197.89 $\pm 0.66 \mathrm{mg} \mathrm{GAE} / \mathrm{g})$, while flavonoids constituted $24.67 \pm 0.15 \%$ of the total polyphenols. Plant-derived phenolic compounds, including flavonoids and phenolic acids, have recently attracted a great deal of interest due to their diverse beneficial effects (29). For instance, Aronia melanocarpa (Rosaceae family) extract contains an abundance of chlorogenic acid (an ester of L-quinic acid and caffeic acid) and rutin (3-rhamnosyl-glucosylquercetin), which was able to prevent ultraviolet (UV) B-induced skin damage by regulating collagen fibers and metalloproteinases in mouse dorsal skin (25). In addition, Rhodomyrtus tomentosa (Myrtaceae family) fruit extract contains numerous phenolic compounds, such as flavonols, anthocyanins and stilbenes (30), and the extract ameliorates bacteria-induced acnes in human skin by reducing inflammatory lesions (31). Furthermore, it is well known that phenolic compounds occur as major ingredients in the commercialized products extracted from Korean and French pine bark (PineXol ${ }^{\circledR}$ and Pycnogenol ${ }^{\circledR}$, respectively) $(12,32,33)$. Pine bark extract has been widely used due to its beneficial attributes. For instance, a previous study demonstrated that oral ingestion of Pycnogenol ${ }^{\circledR}$ protected against skin damage induced by UV irradiation, modulated skin pigmentation and enhanced skin barrier function and extracellular matrix homeostasis (33). In addition, in 2020, Pavlou et al (34) reported that the bark extract of Aleppo pine (Pinus halepensis) protected keratinocytes against cigarette smoke-induced oxidative stress. Of note, a recent study reported that Pinus thunbergii extract ameliorated lipopolysaccharide-induced acute inflammatory responses by regulating oxidative stress (35). In the present study, the total phenols and total flavonoids of the PBE were analyzed; however, liquid chromatography/mass spectrometry analysis, which is frequently used to analyze specific components, was not employed.

The three representative models of alopecia in mice are as follows: i) Genetically modified $\mathrm{C} 3 \mathrm{H} / \mathrm{HeJ}$ mice with spontaneous alopecia; ii) mice with AGA induced by topical application of dihydrotestosterone onto the shaved dorsal skin; and iii) chemotherapy-induced alopecia induced in mice with dorsal hair shaved, followed by intraperitoneal injection of cyclophosphamide (100-125 mg/kg) (36-38). The mouse model of spontaneous alopecia is expensive to develop, in addition to the low success rate (36). Therefore, pilot experiments involving androgenetic and chemotherapy-induced alopecia models were performed by our group and no significant differences in the hair growth rate following shaving of their dorsal hair were noted. The physiological attributes of the hair growth cycle in mice differ from those in humans. In addition, alopecia is not naturally induced in mice. Based on previous studies, although mice do not undergo a vellus-to-terminal hair type switch, or suffer from AGA, they are used as an excellent model system for studying the hair cycle for several reasons $(39,40)$ : i) The first two cycles of mouse hair follicles 

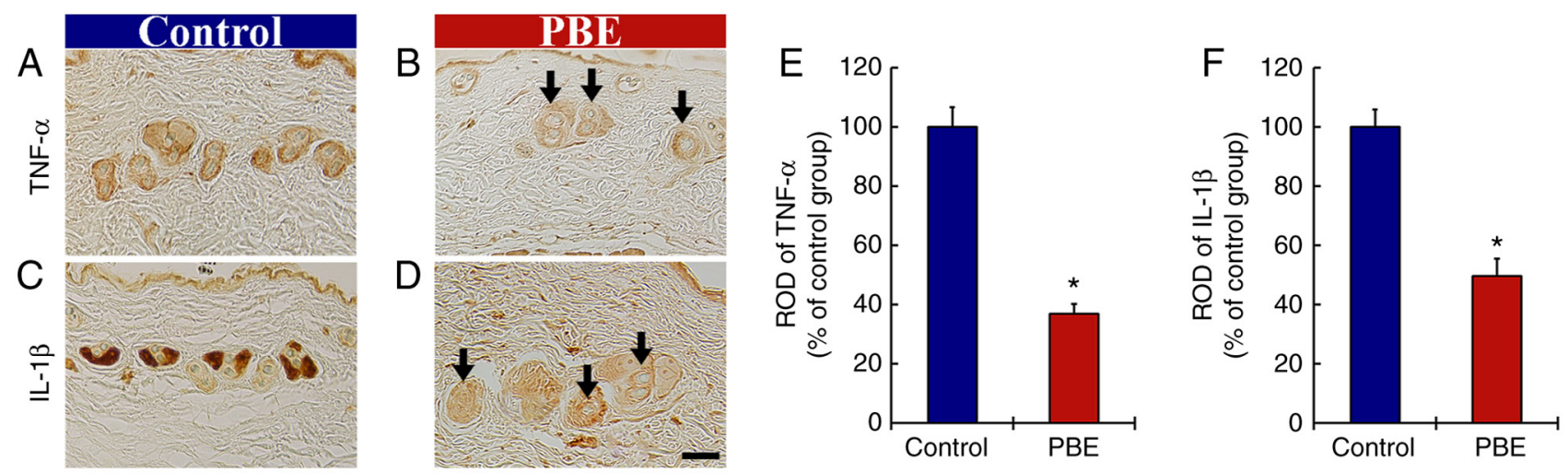

Figure 3. (A-D) Immunohistochemical analysis of TNF- $\alpha$ and IL-1 $\beta$ in the dorsal skin at 17 days after the application. TNF- $\alpha$ in (A) the control group and (B) $2 \%$ PBE group. IL-1 $\beta$ in (C) the control and (D) $2 \%$ PBE group. In the $2 \%$ PBE group, the immunoreactivities of TNF- $\alpha$ and IL-1 $\beta$ were significantly lower (arrows) than those in the control group (scale bar, $50 \mu \mathrm{m}$ ). RODs of (E) TNF- $\alpha$ and (F) IL-1 $\beta$ in the dorsal skin at 17 days after the application. The bars indicate the means \pm standard error of the mean ( $\mathrm{n}=10$ in each group). ${ }^{\mathrm{P}}<0.05$ vs. control group. PBE, Pinus thunbergii bark extract; ROD, relative optical density.
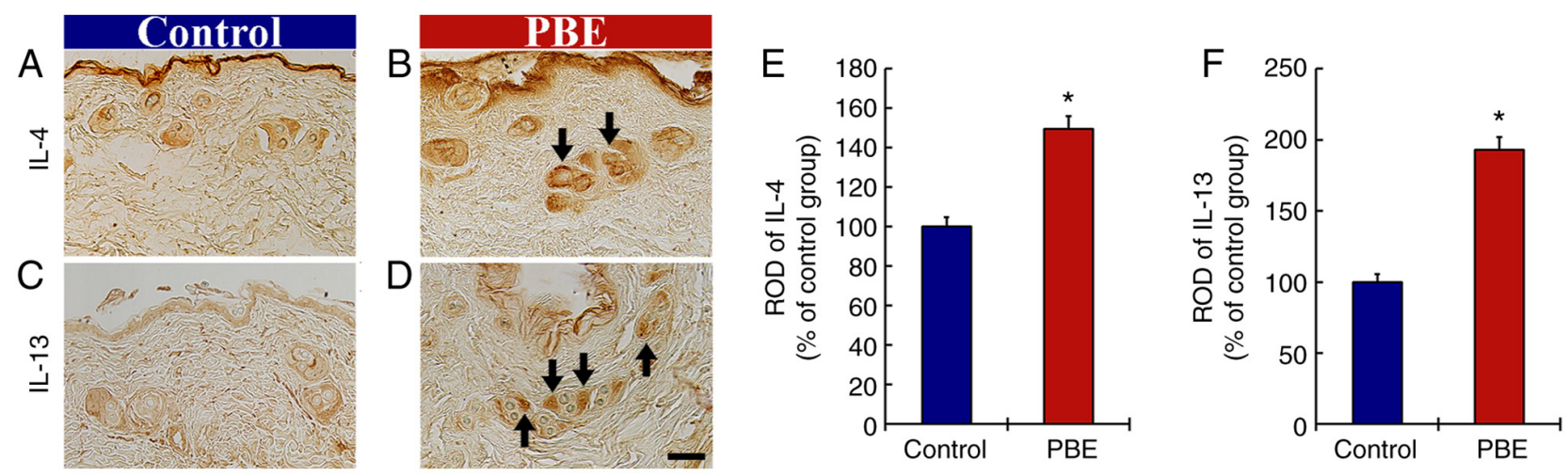

Figure 4. (A-D) Immunohistochemical analysis of IL-4 and IL-13 in the dorsal skin at 17 days after the application. IL-4 in (A) the control group and (B) $2 \%$ PBE group. IL-13 in (C) the control and (D) $2 \%$ PBE group. In the $2 \%$ PBE group, the immunoreactivities of IL- 4 and IL-13 were significantly increased (arrows) as compared with those in the control group (scale bar, $50 \mu \mathrm{m}$ ). RODs of (E) IL-4 and (F) IL-13 in the dorsal skin at 17 days after the application. The bars indicate the means \pm standard error of the mean ( $\mathrm{n}=10$ in each group). ${ }^{*} \mathrm{P}<0.05$ vs. control group. PBE, Pinus thunbergii bark extract; ROD, relative optical density.
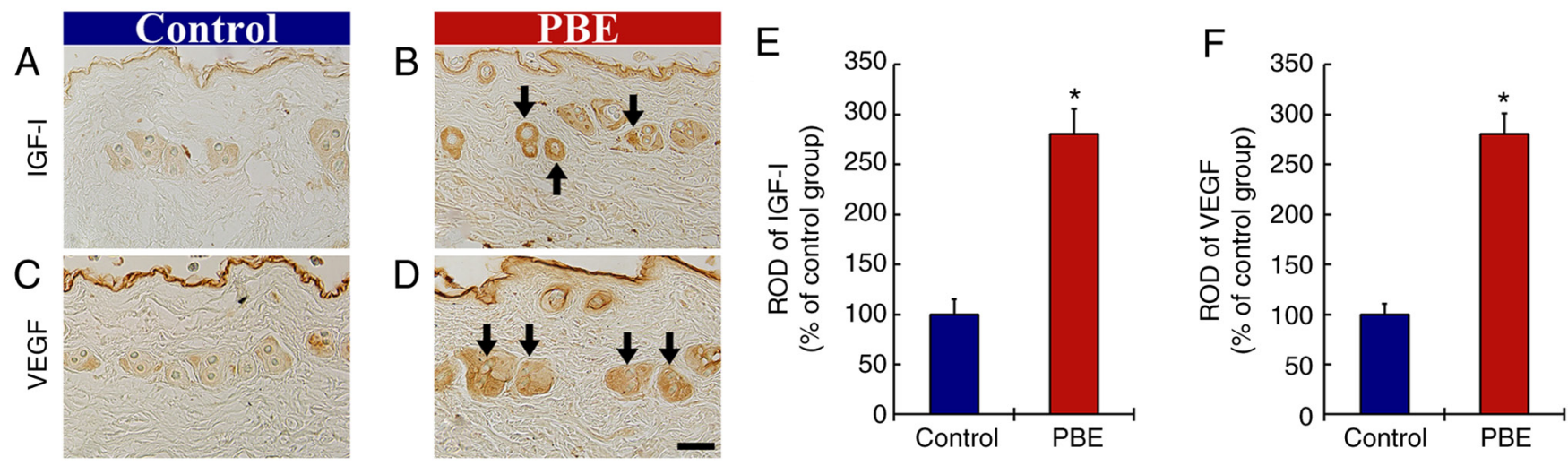

Figure 5. (A-D) Immunohistochemical analysis of IGF-I and VEGF in the dorsal skin at 17 days after the application. IGF-I in (A) the control group and (B) 2\% PBE group. VEGF in (C) the control and (D) 2\% PBE group. In the 2\% PBE group, the immunoreactivities of IGF-I and VEGF were significantly increased (arrows) as compared with those in the control group (scale bar, $50 \mu \mathrm{m}$ ). RODs of (E) IGF-I and (F) VEGF in the dorsal skin at 17 days after the application. The bars indicate the means \pm standard error of the mean ( $\mathrm{n}=10$ in each group). ${ }^{*} \mathrm{P}<0.05$ vs. control group. PBE, Pinus thunbergii bark extract; ROD, relative optical density; IGF, insulin-like growth factor.

are synchronized, while in humans, neighboring follicles cycle independently of each other when biopsy is taken; ii) the hair cycle of mice is short ( $\sim 3$ weeks); by contrast, human scalp hairs have a cycle time of several years and even that of vellus hairs takes months. Therefore, the short-synchronized hair cycle allows hair follicles to be harvested and examined 
at specific time-points in the cycle; and iii) the stages of the hair cycle have been well characterized in the mouse. Anagen is subdivided morphologically into six and catagen into eight different stages $(39,40)$. In this regard, the current model does not completely reflect the pathophysiology of human alopecia. Of note, the hair growth-promoting effects of various treatments have been assessed and for this, the telogen phase was induced by shaving dorsal hair in mice $(3,41-43)$. Based on these experimental data, shaving dorsal hair is regarded as acceptable to assess the hair growth-promoting effect.

In the present study, to investigate the hair growth-promoting effect of PBE, the shaved dorsal skin of mice was treated with DW or 1, 2 and 4\% PBE. The results revealed significant hair growth promotion at 17 days of topical application of 2 and 4\% PBE. However, no differences in cellular distribution and morphology of the dorsal skin tissue were detected following saline or PBE treatment. Accordingly, the optimal dose of PBE to effectively facilitate hair growth was determined as $2 \%$ PBE.

Inflammatory phenomena are increasingly being recognized as an integral part of the progress of AGA $(44,45)$. In AGA, the inflammatory response associated with connective tissue remodeling leads to permanent hair loss (45). In addition, TE, which is another cause of diffuse hair loss, is triggered by stress, shock or traumatic events $(46,47)$. In AGA, scalp biopsies from both male and female patients exhibited follicular micro-inflammation and lymphocytic folliculitis following exposure to immunological stress $(44,48)$. In addition, biopsies of non-scarring alopecia had inflammation in hair follicles in $>20 \%$ of cases (49). A previous study by our group demonstrated the hair growth-promoting effect of Angelica gigas Nakai (Umbelliferae family) root extract mediated via regulating inflammatory cytokines (3). In that study, the regulated pro- and anti-inflammatory cytokines, which were controlled by topical application with root extract of Angelica gigas Nakai, were attributed to upregulation of high-mobility group box 1 (HMGB1) protein, which is an inflammatory modulator of the peri-follicular regions. Furthermore, in 2019, Hwang et al (11) reported that HMGB1 modulated prostaglandin E2 $\left(\mathrm{PGE}_{2}\right)$ metabolism, which was closely associated with inflammatory response, and represented a potential therapeutic target in alopecia. In addition, a precedent study reported that IL-4 enhanced the hair cycle/growth in a mouse model of hapten-induced contact hypersensitivity (50). Furthermore, the previous study by our group indicated that the enhancement of both IL-4 and IL-13 contributed to the hair growth-promoting effect (3). Based on these studies, the effects may be related to the upregulation of IL-4 and IL-13, which may have a hair growth-promoting effect.

Based on the preliminary results, 2\% PBE was applied to the dorsal skin and a significant reduction in the levels of pro-inflammatory cytokines (TNF- $\alpha$ and IL-1 $\beta$ ) and an apparent increase in anti-inflammatory cytokines (IL-4 and IL-13) was observed in the hair follicles. Pro-inflammatory cytokines, which are produced by activated immune cells, induce advanced inflammatory response (51). By contrast, anti-inflammatory cytokines act as antagonists of pro-inflammatory cytokines by suppressing their expression and inhibiting their receptors (52). The present study did not investigate the potential active ingredient(s) of
PBE. However, accumulating data indicate that maritime pine bark extract contains high levels of procyanidins and proanthocyanidins $(18,53,54)$. Based on previous studies, the bark extract of Pinus thunbergii, which is a Korean maritime pine, may also contain procyanidins and proanthocyanidins. In addition, it has been reported that these two components exert anti-inflammatory activity $(55,56)$. Taken together, the phenolic compounds including flavonoids contained in the PBE contribute to excellent anti-inflammatory effects $(57,58)$.

In addition, the results of the present study suggested that the immunoreactivity of IGF-I and VEGF was significantly increased in the hair follicles at 17 days of PBE treatment. Accumulating experimental evidence demonstrated that growth factors, such as IGF-I, VEGF, fibroblast growth factor and epidermal growth factor, stimulate hair growth in vitro and in vivo (10,59-61). Among these growth factors, IGF-I, a potent mitogen assisting cell growth in diverse types of tissues and cells, potently stimulates hair growth. For instance, exogenous IGF-I promotes hair shaft elongation in human hair follicle cells by increasing platelet-derived growth factor, which induces anagen phase in the hair growth cycle (59). VEGF, a growth factor stimulating vasculogenesis and angiogenesis, also facilitates hair growth by providing nutrients to the hair follicles (62). In 2001, Yano et al (10) reported that hair follicle-derived VEGF enhanced perifollicular angiogenesis and increased the number and size of hair follicles in mouse dorsal skin. Furthermore, in 2020, Ahn et al (8) reported that topical application with a mixture of Platycladus orientalis (L.) Franco (Cupressaceae family) leaf extract and $\alpha$-terpineol promoted hair growth in mouse dorsal skin by elevating both IGF-I and VEGF levels in the hair follicles. Taken together, the phenolic compounds including flavonoids present in the PBE were indicated to contribute to hair growth promotion by increasing IGF and VEGF.

In the present study, no in vitro experiments were performed due to technical limitations and no western blot analysis was performed. Therefore, in the future, the mechanisms of the hair growth-promoting effect of $\mathrm{PBE}$ will be investigated using in vitro approaches and western blot analysis and identify a dissolvent that is harmless to the skin and may enhance the hair growth-promoting effect of PBE. In addition, the results of the present study revealed elevated VEGF following topical application of PBE. However, the degrees of vasodilation and angiogenesis were not assessed; therefore, it is suggested that PBE application-induced changes in vasodilation and angiogenesis require to be examined in the future.

In conclusion, the experimental results of the present study suggested that PBE contained polyphenols $(197.89 \pm 0.66 \mathrm{mg}$ GAE/g) and flavonoids (48.82 $\pm 0.14 \mathrm{mg} \mathrm{QE} / \mathrm{g})$, which exerted strong anti-inflammatory activity. A hair growth-promoting effect in mouse dorsal skin was achieved with 1,2 and 4\% PBE, and $2 \%$ PBE effectively promoted hair growth at 17 days of application. To investigate the mechanisms of the hair growth promotion, changes in inflammatory cytokines and growth factors in the hair follicles were immunohistochemically analyzed following treatment with $2 \%$ PBE, revealing that PBE treatment modulated inflammatory responses by reducing the levels of pro-inflammatory cytokines (TNF- $\alpha$ and IL-1 $\beta$ ) and enhancing the levels of anti-inflammatory cytokines (IL-4 and IL-13). Furthermore, certain growth factors (IGF-I and 
VEGF) were significantly increased in the hair follicles. Based on these results, it is strongly suggested that $2 \%$ PBE may be utilized in applications targeting hair growth promotion or hair loss prevention.

\section{Acknowledgements}

The authors would like to express their appreciation to Mr. Seung Uk Lee (Department of Neurobiology, School of Medicine, Kangwon National University) and Ms. Hyun Sook Kim (Department of Biomedical Science and Research Institute for Bioscience and Biotechnology, Hallym University) for their technical help with this work.

\section{Funding}

This work was supported by the Basic Science Research Program through the National Research Foundation (NRF) of Korea funded by the Ministry of Education (grant no. NRF-2019R1A6 A1A11036849) and by the Agriculture, Food and Rural Affairs Convergence Technologies Program for Educating Creative Global Leader (grant no. 714001-07), Ministry of Agriculture, Food and Rural Affairs.

\section{Availability of data and materials}

All data produced and analyzed in the current study are available from the corresponding author on reasonable request.

\section{Authors' contributions}

YH and TKL wrote the draft of the manuscript. JCL, DWK, SYC, MHW and SSK were responsible for experimental conceptualization, data curation and data analysis. YH, TKL, HS, JKH, JWL and JDK performed experiments and data collection and data analysis. TKL and MHW confirm the authenticity of the raw data. SYC, MHW and SSK reviewed and edited the manuscript and acquired funding. All authors read and approved the final manuscript.

\section{Ethics approval and consent to participate}

The experimental procedures using the experimental animals strictly adhered to the 'Current international laws and policies' in the 'Guide for the Care and Use of Laboratory Animals' (24) and approved by the Institutional Animal Care and Use Committee at Kangwon National University (Chuncheon, Republic of Korea; approval no. KW-200114-1; approval date, Jan. 22, 2020).

\section{Patient consent for publication}

Not applicable.

\section{Competing interests}

The authors declare that they have no competing interests.

\section{References}

1. Hunt $\mathrm{N}$ and McHale S: The psychological impact of alopecia. BMJ 331: 951-953, 2005.
2. Liu F,Miao Y,LiX,QuQ,Liu Y,LiK,Feng Cand HuZ:The relationship between self-esteem and hair transplantation satisfaction in male androgenetic alopecia patients. J Cosmet Dermatol: Dec 23, 2018 (Epub ahaed of print). doi: 10.1111/jocd.12839, 2018.

3. Lee TK, Kim B, Kim DW, Ahn JH, Sim H, Lee JC, Yang GE Her Y, Park JH, Kim HS, et al: Effects of decursin and Angelica gigas nakai root extract on hair growth in mouse dorsal skin via regulating inflammatory cytokines. Molecules 25: 3697, 2020.

4. Darwin E, Hirt PA, Fertig R, Doliner B, Delcanto G and Jimenez JJ: Alopecia areata: Review of epidemiology, clinical features, pathogenesis, and new treatment options. Int J Trichology 10: 51-60, 2018.

5. Phillips TG, Slomiany WP and Allison R: Hair loss: Common causes and treatment. Am Fam Physician 96: 371-378, 2017.

6. Guo H, Cheng Y, Shapiro J and McElwee K: The role of lymphocytes in the development and treatment of alopecia areata. Exp Rev Clin Immunol 11: 1335-1351, 2015.

7. Carroll JM, Crompton T, Seery JP and Watt FM: Transgenic mice expressing IFN-gamma in the epidermis have eczema, hair hypopigmentation, and hair loss. J Invest Dermatol 108: 412-422, 1997.

8. Ahn JH, Park YE, Kim B, Park CW, Sim TH, Lee TK, Lee JC, Park JH, Kim JD, Lee HS and Won MH: Hair growth is promoted in mouse dorsal skin by a mixture of Platycladus orientalis (L.) franco leaf extract and alpha-terpineol by increasing growth factors and wnt $3 / \beta$-catenin. Natural Product Communications 15 : 1934578X20951433, 2020.

9. Panchaprateep R and Asawanonda P: Insulin-like growth factor-1: Roles in androgenetic alopecia. Exp Dermatol 23: 216-218, 2014.

10. Yano K, Brown LF and Detmar M: Control of hair growth and follicle size by VEGF-mediated angiogenesis. J Clin Invest 107: 409-417, 2001.

11. Hwang JH, Chu H, Ahn Y, Kim J and Kim DY: HMGB1 promotes hair growth via the modulation of prostaglandin metabolism. Sci Rep 9: 6660, 2019.

12. Kim JW, Im S, Jeong HR, Jung YS, Lee I, Kim KJ, Park SK and Kim DO: Neuroprotective effects of Korean red pine (Pinus densiflora) bark extract and its phenolics. J Microbiol Biotechnol 28: 679-687, 2018.

13. Lim WXJ, Chepulis L, von Hurst P, Gammon CS and Page RA: An acute, placebo-controlled, single-blind, crossover, dose-response, exploratory study to assess the effects of New Zealand pine bark extract $\left(\right.$ Enzogenol $\left.^{\circledR}\right)$ on glycaemic responses in healthy participants. Nutrients 12: 497, 2020.

14. Dridi W and Bordenave N: Pine bark phenolic extracts, current uses, and potential food applications: A review. Curr Pharm Design 26: 1866-1879, 2020.

15. Kim KD, Kim HJ, Park KR, Kim SM, Na YC, Shim BS, Kim SH, Choi SH, Ahn KS, Ryu SH, et al: Pinexol inhibits in vitro inflammatory biomarkers by blocking NF- $\kappa \mathrm{B}$ signaling pathway and protects mice from lethal endotoxemia. Orient Pharm Exp Med 11: 61-70, 2011.

16. Lee YJ, Han OT, Choi HS, Lee BY, Chung HJ and Lee OH: Antioxidant and anti-adipogenic effects of PineXol ${ }^{\circledR}$. Korean J Food Sci Technol 45: 97-103, 2013.

17. Marini A, Grether-Beck S, Jaenicke T, Weber M, Burki C, Formann P, Brenden H, Schönlau F and Krutmann J: Pycnogenol ${ }^{\circledR}$ effects on skin elasticity and hydration coincide with increased gene expressions of collagen type I and hyaluronic acid synthase in women. Skin Pharmacol Physiol 25: 86-92, 2012.

18. Kim B, Lee TK, Park CW, Kim DW, Ahn JH, Sim H, Lee JC, Yang GE, Kim JD, Shin MC, et al: Pycnogenol ${ }^{\circledR}$ supplementation attenuates memory deficits and protects hippocampal CA1 pyramidal neurons via antioxidative role in a gerbil model of transient forebrain ischemia. Nutrients 12: 2477, 2020.

19. Plonka PM, Michalczyk D, Popik M, Handjiski B, Slominski A and Paus R: Splenic eumelanin differs from hair eumelanin in C57BL/6 mice. Acta Biochim Pol 52: 433-441, 2005.

20. Datta K, Singh AT, Mukherjee A, Bhat B, Ramesh B and Burman AC: Eclipta alba extract with potential for hair growth promoting activity. J Ethnopharmacol 124: 450-456, 2009.

21. Adachi T, Kobayashi T, Sugihara E, Yamada T, Ikuta K, Pittaluga S, Saya H, Amagai M and Nagao K: Hair follicle-derived IL-7 and IL-15 mediate skin-resident memory T cell homeostasis and lymphoma. Nat Med 21: 1272-1279, 2015.

22. Duval B and Shetty K: The stimulation of phenolics and antioxidant activity in pea (Pisum sativum) elicited by genetically transformed anise root extract. J Food Biochem 25: 361-377, 2001 
23. Sato M, Ramarathnam N, Suzuki Y, Ohkubo T, Takeuchi M and Ochi H: Varietal differences in the phenolic content and superoxide radical scavenging potential of wines from different sources. J Agric Food Chem 44: 37-41, 1996.

24. Council NR: Guide for the care and use of laboratory animals. 2010.

25. Her Y, Lee TK, Kim JD, Kim B, Sim H, Lee JC, Ahn JH, Park JH, Lee JW, Hong J, et al: Topical application of aronia melanocarpa extract rich in chlorogenic acid and rutin reduces UVB-induced skin damage via attenuating collagen disruption in mice. Molecules 25: 4577, 2020.

26. Carpenter JW: Exotic Animal Formulary-eBook. Elsevier Health Sciences, 2012. https://evolve.elsevier.com/cs/product/97803234 98029?role=student

27. Castro MD: A hematoxylin-eosin phloxine stain for tissues embedded in glycol methacrylate. J Histotechnology 8: 23-24, 1985.

28. Boisvert WA, Yu M, Choi Y, Jeong GH, Zhang YL, Cho S, Choi C, Lee S and Lee BH: Hair growth-promoting effect of Geranium sibiricum extract in human dermal papilla cells and C57BL/6 mice. BMC Complement Altern Med 17: 109, 2017.

29. Zhang L, Ravipati AS, Koyyalamudi SR, Jeong SC, Reddy N, Smith PT, Bartlett J, Shanmugam K, Münch G and Wu MJ: Antioxidant and anti-inflammatory activities of selected medicinal plants containing phenolic and flavonoid compounds. J Agric Food Chem 59: 12361-12367, 2011

30. Lai TN, Andre C, Rogez H, Mignolet E, Nguyen TB and Larondelle Y: Nutritional composition and antioxidant properties of the sim fruit (Rhodomyrtus tomentosa). Food Chem 168 410-416, 2015

31. Gervason S, Metton I, Gemrot E, Ranouille E, Skorski G, Cabannes M, Berthon JY and Filaire E: Rhodomyrtus tomentosa fruit extract and skin microbiota: A focus on C.acnes phylotypes in acne subjects. Cosmetics 7: 53, 2020.

32. Mulek M, Seefried L, Genest F and Högger P: Distribution of constituents and metabolites of maritime pine bark extract $\left(\right.$ Pycnogenol $\left.^{\circledR}\right)$ into serum, blood cells, and synovial fluid of patients with severe osteoarthritis: A randomized controlled trial. Nutrients 9: 443, 2017.

33. Rohdewald P: A review of the French maritime pine bark extract (Pycnogenol), a herbal medication with a diverse clinical pharmacology. Int J Clin Pharmacol Ther 40: 158-168, 2002.

34. Pavlou P, Antoniadou I, Peraki A, Vitsos A, Dallas P, Mostratos D, Deliconstantinos G, Papaioannou G, Grando SA and Rallis M: Protective effects of Pinus halepensis bark extract and nicotine on cigarette smoke-induced oxidative stress in keratinocytes. In Vivo 34: 1835-1843, 2020.

35. Yoon CJ, Choi WS, Kang HS, Kim HJ, Lee WT, Lee JS, Lee S, Son SY, Lee CH, Sohn UD and Lee JY: Pinus thunbergii parl. Extracts reduce acute inflammation by targeting oxidative stress. Evid Based Complement Alternat Med 2021: 7924645, 2021.

36. Shin JM, Choi DK, Sohn KC, Koh JW, Lee YH, Seo YJ, Kim CD, Lee JH and Lee Y: Induction of alopecia areata in $\mathrm{C} 3 \mathrm{H} / \mathrm{HeJ}$ mice using polyinosinic-polycytidylic acid (poly[I:C]) and interferon-gamma. Sci Rep 8: 12518, 2018.

37. Zhu HL, Gao YH, Yang JQ, Li JB and Gao J: Serenoa repens extracts promote hair regeneration and repair of hair loss mouse models by activating TGF- $\beta$ and mitochondrial signaling pathway. Eur Rev Med Pharmacol Sci 22: 4000-4008, 2018.

38. Chen SS, Zhang Y, Lu QL, Lin Z and Zhao Y: Preventive effects of cedrol against alopecia in cyclophosphamide-treated mice. Environ Toxicol Pharmacol 46: 270-276, 2016.

39. Porter RM: Mouse models for human hair loss disorders. J Anat 202: 125-131, 2003

40. Muller-Rover S, Handjiski B, van der Veen C, Eichmüller S, Foitzik K, McKay IA, Stenn KS and Paus R: A comprehensive guide for the accurate classification of murine hair follicles in distinct hair cycle stages. J Invest Dermatol 117: 3-15, 2001.

41. Ahn S, Lee JY, Choi SM, Shin Y and Park S: A mixture of tocopherol acetate and L-menthol synergistically promotes hair growth in C57BL/6 mice. Pharmaceutics 12: 1234, 2020.

42. Jiang H, Yamamoto S and Kato R: Induction of anagen in telogen mouse skin by topical application of FK506, a potent immunosuppressant. J Invest Dermatol 104: 523-525, 1995.
43. Xiao L, Zhang X, Chen Z, Li J, Li B and Li L: Molecular pathways involved in promoting activity of timosaponin BII on hair growth in C57BL/6 mice. Biomed Res Int 2020: 9451596, 2020.

44. Magro CM, Rossi A, Poe J, Manhas-Bhutani S and Sadick N: The role of inflammation and immunity in the pathogenesis of androgenetic alopecia. J Drugs Dermatol 10: 1404-1411, 2011.

45. Trüeb RM: Molecular mechanisms of androgenetic alopecia. Exp Gerontol 37: 981-990, 2002.

46. Arck PC, Handjiski B, Peters EM, Peter AS, Hagen E, Fischer A, Klapp BF and Paus R: Stress inhibits hair growth in mice by induction of premature catagen development and deleterious perifollicular inflammatory events via neuropeptide substance P-dependent pathways. Am J Pathol 162: 803-814, 2003.

47. Malkud S: A hospital-based study to determine causes of diffuse hair loss in women. J Clin Diagn Res 9: WC01-WC04, 2015.

48. El-Domyati M, Attia S, Saleh F and Abdel-Wahab H: Androgenetic alopecia in males: A histopathological and ultrastructural study. J Cosmet Dermatol 8: 83-91, 2009.

49. Aslani FA, Esfahani MH and Sepaskhah M: Non-scarring alopecias in Iranian patients: A histopathological study with hair counts. Iran J Pathol 13: 317-324, 2018.

50. Sugita K, Nomura T, Ikenouchi-Sugita A, Ito T, Nakamura M, Miyachi Y, Tokura Y and Kabashima K: Influence of Th2 cells on hair cycle/growth after repeated cutaneous application of hapten. Clin Exp Dermatol 39: 213-215, 2014

51. Zhang JM and An J: Cytokines, inflammation, and pain. Int Anesthesiol Clin 45: 27-37, 2007.

52. Opal SM and DePalo VA: Anti-inflammatory cytokines. Chest 117: 1162-1172, 2000.

53. Park JH, Kim JD, Lee TK, Han X, Sim H, Kim B, Lee JC, Ahn JH, Lee CH, Kim DW, et al: Neuroprotective and anti-inflammatory effects of Pinus densiflora bark extract in gerbil hippocampus following transient forebrain ischemia. Molecules 26: 4592, 2021.

54. Robertson NU, Schoonees A, Brand A and Visser J: Pine bark (Pinus spp.) extract for treating chronic disorders. Cochrane Database Syst Rev 9: CD008294, 2020.

55. Ma X, Wang R, Yu S, Lu G, Yu Y and Jiang C: Anti-inflammatory activity of oligomeric proanthocyanidins via inhibition of NF- $\kappa \mathrm{B}$ and MAPK in LPS-stimulated MAC-T Cells. J Microbiol Biotechnol 30: 1458-1466, 2020.

56. Saldanha AA, do Carmo LF, do Nascimento SB, de Matos NA, de Carvalho Veloso C, Castro AHF, De Vos RCH, Klein A, de Siqueira JM, Carollo CA, et al: Chemical composition and anti-inflammatory activity of the leaves of Byrsonima verbascifolia. J Nat Med 70: 760-768, 2016.

57. Toda K, Tsukayama I, Nagasaki Y, Konoike Y, Tamenobu A, Ganeko N, Ito H, Kawakami Y, Takahashi Y, Miki Y, et al: Red-kerneled rice proanthocyanidin inhibits arachidonate 5-lipoxygenase and decreases psoriasis-like skin inflammation. Arch Biochem Biophys 689: 108307, 2020.

58. Park CH, Min SY, Yu HW, Kim K, Kim S, Lee HJ, Kim JH and Park YJ: Effects of apigenin on RBL-2H3, RAW264.7, and $\mathrm{HaCaT}$ cells: Anti-allergic, anti-inflammatory, and skin-protective activities. Int J Mol Sci 21: 4620, 2020.

59. Ahn SY, Pi LQ, Hwang ST and Lee WS: Effect of IGF-I on hair growth is related to the anti-apoptotic effect of IGF-I and Up-regulation of PDGF-A and PDGF-B. Ann Dermatol 24: 26-31, 2012

60. Lin WH, Xiang LJ, Shi HX, Zhang J, Jiang Lp, Cai Pt, Lin ZL, Lin BB, Huang Y, Zhang HL, et al: Fibroblast growth factors stimulate hair growth through $\beta$-catenin and Shh expression in C57BL/6 mice. Biomed Res Int 2015: 730139, 2015.

61. Lin Y, Liu C, Zhan X, Wang B, Li K and Li J: Jagged1 and epidermal growth factor promoted androgen-suppressed mouse hair growth in vitro and in vivo. Front Pharmacol 10: 1634, 2019

62. Gnann LA, Castro RF, Azzalis LA, FederD, Perazzo FF, Pereira EC, Rosa PCP, Junqueira VBC, Rocha KC, Machado CA, et al: Hematological and hepatic effects of vascular epidermal growth factor (VEGF) used to stimulate hair growth in an animal model. BMC Dermatol 13: 15, 2013.

This work is licensed under a Creative Commons Attribution-NonCommercial-NoDerivatives 4.0 International (CC BY-NC-ND 4.0) License. 